
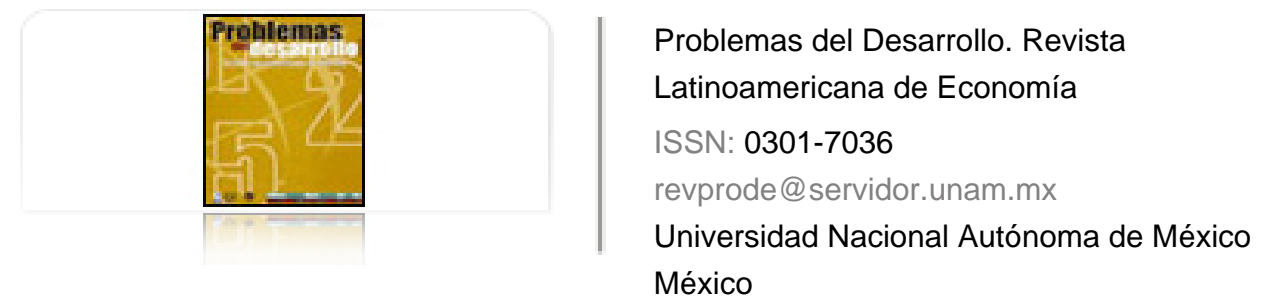

González García, Juan

LA EVOLUCIÓN DEL EMPLEO EN CHINA: ENTRE EL DISFRAZ DEL PLENO EMPLEO Y LOS PROBLEMAS DE OFERTA DE TRABAJO PARA LA POBLACIÓN ECONÓMICAMENTE ACTIVA

Problemas del Desarrollo. Revista Latinoamericana de Economía, vol. 34, núm. 134, 2003, pp. 75-94

Universidad Nacional Autónoma de México

Distrito Federal, México

Disponible en: http://www.redalyc.org/articulo.oa?id=11825944005

Cómo citar el artículo

- Número completo

- Más información del artículo

Página de la revista en redalyc.org

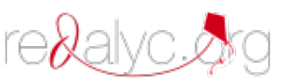

Sistema de Información Científica

Red de Revistas Científicas de América Latina, el Caribe, España y Portugal Proyecto académico sin fines de lucro, desarrollado bajo la iniciativa de acceso abierto 


\title{
LA EVOLUCIÓN DEL EMPLEO EN CHINA: ENTRE EL DISFRAZ DEL PLENO EMPLEO Y LOS PROBLEMAS DE OFERTA DE TRABAJO PARA LA POBLACIÓN ECONÓMICAMENTE ACTIVA
}

\author{
Juan González García*
}

Fecha de recepción: 13 de mayo de 2003. Fecha de autorización: 3 de diciembre de 2003.

\begin{abstract}
Resumen
En este artículo se analiza la política de empleo en China, particularmente la desarrollada en la última década del siglo Xx. Demostramos que, si bien la política de empleo se ha orientado a resolver los problemas de la apertura económica y modernización de las empresas del Estado, los retos que la tasa de crecimiento de la población económicamente activa le formula (PEA) determinarán, en gran medida, el éxito o fracaso de la estrategia de apertura externa pues ésta, desde sus inicios fue concebida para atender, entre otros problemas, las necesidades de dotación de empleo y de ingreso de la PEA. Concluye, con una parte prospectiva en torno a los obstáculos que tendrá que vencer la República Popular China para garantizar la dotación de empleo a su población..
\end{abstract}

Palabras clave: empleo, sistema de contratos, empresas del Estado, reforma económica, mercado, productividad.

\begin{abstract}
This article analyzes the Chinese employment policy, particularly in the last decade of the twentieth century. It also shows that although the employment policy has aimed to solve the problems of economic liberalization and modernization of public companies, the challenges posed by the growth rate of the economically active population (EAP) will to a large extent determine the success or failure of the strategy to open up since from its conception it was meant to respond to the need of providing the EAP with employment and income, among other needs. It concludes with a prospective of the obstacles the People's Republic of China will have to overcome in order to warrantee employment for the Chinese population.
\end{abstract}

Key words: employment, contracting system, public entreprises, economic reform, market, productivity.

* Universidad de Colima. 


\section{Résumé}

Cet article analyse la politique de l'emploi en Chine, en particulier celle qui a été développée durant la dernière décennie du xxème siècle. Nous y démontrons que, bien que la politique de l'emploi se soit orientée vers la résolution des problèmes de l'ouverture économique et de la modernisation des entreprises de l'État, les défis qui lui sont lancés par le taux de croissance de la population économiquement active (PEA) vont déterminer dans une grande mesure le succès ou l'échec de la stratégie d'ouverture externe. En effet, celle-ci a été conçue dès le départ pour répondre, entre autres problèmes, aux besoins de dotation d'emploi et de revenu à la PEA. Il conclut par une partie prospective qui traite des obstacles que devra surmonter la République Populaire Chinoise pour garantir la dotation d'emploi à sa population.

Mots-cléfs: emploi, système de contrats, entreprises de l'État, réforme économique, marché, productivité.

\section{Resumo}

Neste artículo é analisada a política de emprego na China, principalmente a desenvolvida na última década do século xx. Demonstramos que, embora as política de emprego tenha sido orientada para resolver os problemas da abertura econômica e a modernização das empresas do Estado, os desafios que a taxa de crescimento da população economicamente ativa coloca (PEA) determinarão, em grande medida, o êxito ou fracasso da estratégia de abertura externa já que esta, desde o começo, foi concebida para atender, entre outros problemas, as necessidades de emprego e de renda da PEA. Conclui com uma parte prospectiva sobre os obstáculos que terá que vencer a República Popular da China para garantir emprego à sua população.

Palavras chave: emprego, sistema de contratos, empresas do estado, reforma econômica, mercado, produtividade. 


\section{Introducción}

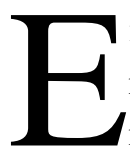

n un escenario internacional caracterizado por la fuerte competencia entre las naciones — desarrolladas y subdesarrolladas - por atraer capital para complementar el ahorro interno y estimular así sus procesos de crecimiento sano y sostenido, la generación de empleo tanto en las empresas como en los gobiernos de dichos países se está convirtiendo en uno de los principales catalizadores para medir la eficiencia de las políticas económicas en lo relativo al desarrollo económico.

Dotar de empleo a la población en edad de trabajar, preparada o no para ello, es actualmente uno de los principales retos de la comunidad internacional y nacional, para con la población. ${ }^{1}$ De hecho, como lo reconoce la Organización Internacional del Trabajo (OIT, 2000:7), es uno de los más graves problemas que debe resolver la humanidad. Sobre todo si se toma en cuenta que, en las dos últimas décadas del siglo xx, la tendencia de la economía internacional, basada cada vez más en tecnologías ahorradoras de mano de obra (Aaronson y Housinger, 1999) ha sido la de expulsar, más que absorber, fuerza de trabajo de una población que, año con año, se incorpora al mundo laboral.

De acuerdo con los últimos reportes de la oIT, entre 1998 y 2001, el empleo ha seguido la desaceleración de la economía mundial iniciada a principios de 2000, presentándose en el mercado laboral una tendencia hacia la contracción, circunstancia que ha arrojado una cifra en 2001 de entre 160 y 200 millones de trabajadores desempleados en el mundo y entre 700 y 800 millones de subempleados. Esta situación real, paradójicamente contradice algunas cifras reportadas por muchos países en sus estadísticas, las cuales registran tasas de desempleo de entre 2.5 y 4.5\%. Lo más grave es que para el año 2010 se estima que se requerirán 460 millones de empleos (orT, 2001:2). Estas cifras de desempleo, subempleo y estimaciones de demanda de trabajo son de gran magnitud, desde cualquier ángulo que se les aborde.

Ante este panorama mundial, resulta interesante saber qué están haciendo los países para dar respuesta a las crecientes necesidades de empleo de su población. En particular, se analizará el caso de la República Popular China (RPC). Este país, el más poblado del

1 La variable empleo está asociada con su contraparte, desempleo. De hecho, no se pueden tratar independientemente una de otra. Por ese motivo, aunque nuestro interés principal se centra en la manera cómo China está resolviendo su problema de empleo, invariablemente nos referiremos a la otra cara de la moneda por mantener ambas una relación directa. 
mundo, con 1276.8 miles de millones de habitantes y una PEA de alrededor de 750 millones de personas en 2002, constituye un termómetro importante en sí mismo para saber en qué medida se ajusta o no al comportamiento internacional del mercado de trabajo.

El objetivo de este artículo es hacer un breve análisis en torno a la respuesta que China está dando a la demanda de su población para acceder a un empleo formal y analizar la estructura y comportamiento de éste, particularmente en la fase de su apertura externa y de los retos que enfrenta para satisfacer dichas demandas laborales, a partir de su regresoadmisión (binomio que veremos más adelante) al Acuerdo General sobre Comercio y Aranceles (GATT, por sus siglas en inglés), hoy Organización Mundial de Comercio (OMC), en diciembre de 2001.

La forma en la que estructuraremos nuestro análisis se conformará por cinco partes: la primera analizará la política y evolución del empleo bajo el socialismo de Mao Zedong; la segunda, presentará las modificaciones en la política de empleo a partir de la reforma económica de 1978; la tercera parte, la estructura y evolución del empleo en China; la cuarta parte, se abocará a analizar la vinculación entre políticas de empleo y bienestar de la población, en tanto expresión directa del éxito o fracaso de la política de desarrollo y empleo, y; finalmente, se estudiará el desafío que implicará para China su admisiónregreso al GATT-OMC. Habrá una parte de conclusiones generales en torno a la problemática analizada en la que expondremos algunos puntos de vista.

\section{Evolución del empleo durante el socialismo: 1949-1978}

Durante el periodo que abarca la etapa del socialismo en China (1949-1978), se estableció un modelo de desarrollo económico diseñado, regido y aplicado por el Estado, como propietario de los medios de producción, fundamentado en la industria pesada (Eckstein, 1977). Durante un lapso de treinta años el Estado implantó, tanto en el sector industrial —liderado por las empresas propiedad del Estado (EPE) — como en el sector agrícola — representado por la figura de la Comuna Popular (CP) - una política de empleo semejante, valga la analogía, con el pleno empleo keynesiano. ${ }^{2}$ Aunque en este caso, debemos decir una política laboral de nulo desempleo en la que cada individuo tenía garantizado, como derecho constitucional, el acceso al empleo, ya fuera en el área rural o en la industria estatal urbana, respondiera o no a las expectativas del trabajador o a las necesidades de la empresa. Finalmente, el Estado asume y garantiza este derecho constitucional.

2 Es decir, mediante una perspectiva teórica y categorías de análisis diferentes, la finalidad de la política de desarrollo económico en China dotaba de empleo a toda la población en edad de trabajar, independientemente de sus capacidades y aptitudes laborales. El objetivo era emplear el factor abundante en las diferentes esferas de la producción para propiciar la consolidación deuna estructura económica en ciernes. 
Cuadro 1

Evolución del empleo en China, 1952-1978

(decenas de miles de personas)

\begin{tabular}{|c|c|c|c|}
\hline$A \tilde{n} o$ & Población total (A) & Empleo total (B) & $B / A(\%)$ \\
\hline 1952 & 57428 & 20729 & 36.1 \\
\hline 1953 & 58796 & 21364 & 36.3 \\
\hline 1954 & 60266 & 21832 & 36.2 \\
\hline 1955 & 61465 & 22388 & 36.3 \\
\hline 1956 & 62828 & 23018 & 36.6 \\
\hline 1957 & 64653 & 23771 & 36.8 \\
\hline 1958 & 65994 & 26600 & 40.3 \\
\hline $1959 *$ & 67207 & 26173 & 38.9 \\
\hline 1960 & 66207 & 25880 & 39.0 \\
\hline 1961 & 65859 & 25590 & 38.9 \\
\hline 1962 & 67295 & 25910 & 38.5 \\
\hline 1963 & 69172 & 26640 & 38.5 \\
\hline 1964 & 70499 & 27736 & 39.3 \\
\hline 1965 & 72538 & 28670 & 39.5 \\
\hline 1966 & 74542 & 29805 & 40.0 \\
\hline 1967 & 76368 & 30814 & 40.3 \\
\hline 1968 & 78534 & 31915 & 41.0 \\
\hline 1969 & 80671 & 33225 & 41.1 \\
\hline 1970 & 82992 & 34432 & 41.5 \\
\hline 1971 & 85229 & 35620 & 41.8 \\
\hline 1972 & 87177 & 35854 & 41.1 \\
\hline 1973 & 89211 & 36652 & 41.1 \\
\hline 1974 & 90859 & 37369 & 41.1 \\
\hline 1975 & 92420 & 38168 & 41.3 \\
\hline 1976 & 93717 & 38834 & 41.1 \\
\hline 1977 & 94974 & 39377 & 41.5 \\
\hline 1978 & 96259 & 39856 & 41.4 \\
\hline
\end{tabular}

Nota: * Los años 1959-1962 registran un decremento de la población y del empleo, debido a las políticas del Gran Salto Adelante (GSA).

Fuente: Tomado de González, Juan y Liu Xue Dong, "El mercado laboral en China. Situación actual y perspectivas”, Comercio Exterior, núm. 7, vol. 49, p. 629.

Con la intervención y conducción del Estado durante el periodo socialista, la oferta de empleo creció a una tasa promedio de $0.5 \%$, menor a la tasa de crecimiento demográfico de $1.87 \%$ (véase Cuadro 1). Sin embargo, desde un esquema de cero desempleo se otorgó trabajo a $100 \%$ de la fuerza laboral china. La tasa de empleo relacionada con la población total se mantuvo próxima a 39\% de esta última. Los únicos años en que hubo algún problema con la colocación de empleados fue en los del Gran Salto Adelante (GSA), donde incluso, hubo un decremento de la población.

3 En la contabilidad socialista el sector servicios no se consideraba, debido a que sólo se registraba la producción material, aquella que representa la transformación dela naturaleza mediante la incorporación del trabajo vivo, del trabajo de los obreros y campesinos. 
Durante el periodo del socialismo, el Estado determinó, para atender el conflicto del empleo en el mercado laboral, políticas de contratación de trabajadores tanto para la CP como para las $\mathrm{EPE}^{3}$ sujetas a sus objetivos político-ideológicos. Además, aplicó una política de estricto control de los trabajadores (sistema hukou) para prevenir y evitar los flujos migratorios entre las áreas urbanas y rurales (Fang Shan,1996). Sin embargo, pese a esta última política de control, siempre se mantuvo la posibilidad de los flujos laborales, la cual se explicaba por los diferenciales de salario entre la agricultura y la industria —entre el área rural y urbana-, por los servicios sociales y urbanos y por las tendencias naturales de la población a moverse, particularmente en el GSA y de la Revolución Cultural (RC).

Uno de los efectos dañinos de este tipo de políticas propició que los trabajadores fueran colocados por las oficinas de empleo — de acuerdo con criterios políticos y no económicos $y$, peor aún, que fueran colocados, en lo general- sin corresponder a las necesidades específicas de mano de obra por parte de las EPE e inclusive de las CP. Ello derivaría en problemas de rendimiento decreciente del factor trabajo, sobrepoblamiento y desmotivación por parte del trabajador (Muqiao, 1982).

Estos problemas de empleo, sin embargo, eran minimizados, pues el Estado decidió privilegiar el igualitarismo sobre la productividad. Con ello generó desperdicio y desorganización en el trabajo, ya que las CP y las EPE, frecuentemente eran manejadas por cuadros del Partido Comunista Chino (Deleyne, 1976), cuyos dirigentes poco caso hacían de la organización científica del trabajo y más bien lo inhibían (como en las etapas del mercado — 1961-1964 - y la RC — 1966-1969—) $)^{4}$ y castigaban la productividad y la creatividad. Lo anterior provocó ineficiencias, desperdicios y altos costos laborales: las labores que normalmente debería hacer un trabajador, la hacían dos, tres, y hasta cuatro (Fang Shan, 1996).

\section{Reforma económica y apertura externa: política de empleo}

Con la reforma económica iniciada en diciembre de $1978^{5}$ se establecieron las condiciones para que la política de empleo se modificara. En efecto, se implantó el mecanismo de mercado, la descentralización económica, modificaciones jurídico-económicas para incentivar el desarrollo de la economía privada, la introducción del sistema de contratos en la agricultura, industria y comercio exterior, el inicio de la liberación del sistema de

4 Cuando concluyó la RC (1976, aunque oficialmente fue en el año de 1969) se estimaba que existían alrededor de 17 millones de jóvenes esperando trabajo en las ciudades.

5 En este artículo no se analiza ni la evolución de la economía, política y sociedad chinas, no es nuestro objetivo. Romer Cornejo (2001) hace una excelente semblanza de los últimos cincuenta años del socialismo chino y, en particular, trata en detalle las vicisitudes del proceso de cambio político en China desdela muerte de $M$ ao Zedong, el 9 de septiembre de 1976, hasta el arribo al poder de Deng Xiaoping. 
Cuadro 2

Viejo y nuevo sistema de relaciones laborales en China

\begin{tabular}{ll}
\hline \multicolumn{1}{c}{ Viejo o maoísta } & \multicolumn{1}{c}{ Nuevo o reformista } \\
\hline Economía socialista & Mecanismo de mercado \\
Eficiencia tecnológica & Asignación eficiente \\
Relativamente "Roja" & Relativa experiencia \\
Cuadros políticos dirigiendo & Administradores profesionales \\
Empresas propiedad del Estado & Propiedad diversa de empresas \\
Trabajo de por vida & Contratos laborales \\
Asignación del trabajo & Elección de trabajo \\
Asignación de trabajadores & Selección de trabajadores \\
Salario con base en igualitarismo & Salario con base en el cumplimiento \\
Estado de bienestar dentro de casa & Contribución al seguro social \\
Compañías familiares & Mercado asigna acomodo laboral \\
Fortaleza de uniones laborales & Débil representación del trabajador \\
\hline
\end{tabular}

Fuente: Tomado de Zhu y Malcon Warner (2000), "Changing appoaches to employment relations in the People's Republic of China (PRC)", en Greg Basbon et al., Employment Relations in the Asia Pacific (changing approaches), Australia, Allen and Unwin, p. 118.

precios duales y demás condiciones, las cuales sentaron las bases para la transformación del mercado laboral en China, guiado por el Estado.

En el Cuadro 2 observamos el sistema laboral chino, tanto en el periodo socialista como en la reforma económica, posteriormente llamado socialista de mercado. Destaca la institucionalización de un nuevo mercado laboral que inhibe al gobierno como oferente de empleo directo y estimula el mecanismo de mercado para que las empresas lleven a cabo la contratación de cuadros, con criterios de productividad.

Grosso modo, se puede afirmar que con su reforma económica y apertura externa, China aplicó políticas específicas para liberar el mercado laboral y ajustarlo al mecanismo de mercado de una manera gradual. Lo anterior se puede ubicar en algunos años específicos de las dos últimas décadas del siglo xx y principios del siglo xxI, particularmente a raíz del inicio de las negociaciones finales sostenidas con la OMC entre 2000 y 2001:

a) 1980: Introducción del sistema contractual en determinadas actividades: agricultura, industria y comercio, posteriormente, comercio exterior. Para estimular la producción y productividad laboral: incentivos económicos a la producción.

b) 1984: Incorporación de las Empresas Propiedad del Estado, mediante el inicio del proceso de modernización de éstas a la reforma laboral.

c) 1986: Para eliminar la mentalidad del empleo socialista o para toda la vida, se amplió el sistema contractual a todo el país y se liberó la administración interna de las empresas.

d) 1990: Inicio de una nueva etapa: la movilidad del trabajo dentro de la empresa, para optimizar la estructura de la fuerza de trabajo. 
e) 1993: Permiso a las empresas para emplear trabajadores y permitir que los individuos se ocupen en las actividades que ellos decidan libremente, de acuerdo con el mecanismo de mercado.

f) 1995: Establecimiento de un salario mínimo fijado en RMB mensuales, para las diversas regiones.

g) 1996-2000: Diversas medidas que se orientan a permitir la incorporación de los trabajadores rurales a las zonas urbanas y a ampliar la productividad con estímulos.

h) 2002: Liberación mayor a partir de fases decisivas de negociación en el ingreso a la OMC. Eliminación total de controles a los flujos laborales al interior del país.

Durante este periodo de más de dos décadas, que podríamos calificar de aproximación a la normalización occidental del mercado laboral, China ha dado una mayor libertad para que las empresas contraten trabajo (oferta) como para que los trabajadores acepten (demanda) y — siempre acorde a las necesidades laborales-, guiada por el mecanismo de utilidad más que por criterios políticos, ideológicos o asistencialistas.

La política de empleo en la República Popular, con la liberación del mercado laboral y su desregulación, al establecer políticas de alcance nacional y políticas de reempleo para los trabajadores urbanos y rurales, se ajusta a las prácticas internacionales del mundo capitalista y de los países de economía mixta, en los cuales dichas políticas prevalecen desde hace décadas.

En particular, la introducción del sistema de contratos permitió a las empresas agrícolas y de propiedad del Estado, la contratación de trabajadores, acorde a sus necesidades de producción, organización, administración y gestión. En adelante, tanto la empresa como los propios trabajadores se ajustan a las prácticas del mercado y al sistema de contratos laborales que, de acuerdo con Fang Shang (1996), permitieron normar la relación contractual al establecer derechos y obligaciones de trabajadores y contratantes. Estos últimos serían no sólo las empresas del sector estatal, sino las de capital privado, nacional e internacional, bajo los criterios básicos de rentabilidad y productividad de la mano de obra.

A la par de esta liberación del mercado laboral, se estimuló la creación de empresas dedicadas a la contratación, capacitación, selección de personal, para mediar entre trabajadores y empleadores. Con ello, se completó el marco general para la reforma — política de empleo - al mercado laboral chino: Estado (disposiciones, normas, políticas), oferta laboral (empresas públicas y privadas) y demanda de trabajo (PEA y sus diversos grados de calificación).

Desde este esquema, la política de empleo que guía al mercado laboral chino, cada vez menos perturbado por las acciones político-ideológicas del Estado, enfrenta los desafíos de brindar empleo a una PEA creciente y de proveerla de ingresos suficientes. Cabe agregar que, con la reforma, la estructura económica respondió con creces a los nuevos incentivos 
y ello se reflejó en el crecimiento alto y sostenido del PIB (a diferencia de algunos años de la fase del socialismo de Mao Zedong), lo que a su vez, ha permitido la creación de importantes fuentes de empleo, particularmente en los sectores industrial y de servicios, que son los que están liderando la trasformación estructural de la economía china.

\section{Estructura y evolución del empleo en China, 1979-2002}

Si analizamos el comportamiento del empleo en el periodo de la reforma económica china, tenemos que — una vez que se optó por reestructurar las empresas privadas y de otorgarles autonomía tanto a las EPE, para contratar personal, como a las empresas rurales y granjas familiares para invertir en la agroindustria y contratar trabajo- la generación de empleo ha estado, en consecuencia, más asociada a la capacidad, habilidad y destreza del trabajador que a factores políticos o ideológicos. Obviamente, aunque este nuevo sistema no se aplicó de inmediato y no se sustituyó de forma completa — por el mismo gradualismo- el antiguo sistema de contratar trabajo de acuerdo con su productividad, provocó un fuerte shock entre los trabajadores, acostumbrados al anterior régimen de contratación que les garantizaba empleo para toda la vida, de modo independiente de las necesidades reales de la empresa.

Para este cambio, la figura institucional de los contratos laborales ha desempeñado un papel importante, ya que ha redefinido los derechos y obligaciones tanto de empleadores como de empleados, incrementando la movilidad del empleo. Por su parte, los departamentos de trabajo del gobierno fueron, y están siendo, instados a ofrecer servicios laborales como si fueran compañías lucrativas, pero no a la usanza de las antiguas agencias de colocación. Ahora deben desarrollar cursos de capacitación y dirigirse por sí mismas.

Ante este nuevo marco, el panorama del empleo cambió. El trabajo se otorga de acuerdo con las necesidades reales de la empresa, a su productividad marginal y con criterios económicos. Según Rodríguez (1998), al introducir el sistema de contratos en la agricultura e industria, el empleo ha tendido a ser más sofistificado, debido al uso de técnicas y tecnologías, que a su vez, exigen mayor nivel de capacitación y especialización por parte de los trabajadores. Asimismo, está empezando a generar problemas de insuficiencia de mano de obra calificada en los sectores intensivos en el uso de tecnologías ahorradoras de trabajo: automotriz, electrónica, conductores, informática, cibernética, nuevos materiales, biotecnología, nanotecnología, entre otros.

Con la transformación del mercado laboral, desde el punto de vista de la oferta y la demanda, se terminó con el pleno empleo tradicional del socialismo, lo que nosotros llamamos pleno empleo disfrazado. Si bien esta transformación laboral está llevando al second-best laboral en el corto plazo, creemos que en el largo plazo, redundará en mayores beneficios tanto para las empresas como para los trabajadores. Hoy día, si China enfrenta problemas de desempleo, estos corresponden, principalmente, a factores estructura- 
Cuadro 3

Evolución del empleo en China, 1979-2001

(deceneas de miles de personas y porcentaje)

\begin{tabular}{|c|c|c|c|c|c|}
\hline$A \tilde{n} o$ & $\begin{array}{c}\text { Población } \\
\text { total }(A)\end{array}$ & PEA $(B)$ & $\begin{array}{c}\text { PEA } \\
\text { ocupada }(C)\end{array}$ & $(A / C)$ & $\begin{array}{c}\text { Desempleo } \\
(\%)\end{array}$ \\
\hline 1979 & 97542 & 40412 & 39856 & 0.41 & 2.8 \\
\hline 1980 & 98705 & 40890 & 42361 & 0.41 & 2.6 \\
\hline 1981 & 98796 & 42716 & 42661 & 0.43 & 2.5 \\
\hline 1982 & 100260 & 43602 & 43364 & 0.43 & 2.4 \\
\hline 1983 & 102335 & 46707 & 46436 & 0.45 & 2.3 \\
\hline 1984 & 103687 & 48433 & $48 \quad 197$ & 0.46 & 1.9 \\
\hline 1985 & 105110 & 50112 & 49873 & 0.47 & 2.0 \\
\hline 1986 & 106685 & 51546 & 51282 & 0.48 & 2.0 \\
\hline 1987 & 108410 & 53060 & 52783 & 0.49 & 2.0 \\
\hline 1988 & 110160 & 54630 & 54334 & 0.49 & 2.6 \\
\hline 1989 & 111872 & 55707 & 55329 & 0.49 & 2.5 \\
\hline 1990 & 113521 & 64483 & 63904 & 0.56 & 2.3 \\
\hline 1991 & 115081 & 65399 & 64799 & 0.56 & 2.3 \\
\hline 1992 & 116510 & 66184 & 65554 & 0.56 & 2.6 \\
\hline 1993 & 117805 & 67033 & 66373 & 0.56 & 2.8 \\
\hline 1994 & 119180 & 67879 & 67199 & 0.56 & 2.9 \\
\hline 1995 & 120490 & 68737 & 67947 & 0.56 & 3.0 \\
\hline 1996 & 121761 & 69665 & 68850 & 0.57 & 3.1 \\
\hline 1997 & 123012 & 70800 & 69820 & 0.57 & 3.1 \\
\hline 1998 & 124221 & 72087 & 70637 & 0.57 & 3.1 \\
\hline 1999 & 125361 & 72791 & 71394 & 0.57 & 3.1 \\
\hline 2000 & 126583 & 73992 & 72085 & 0.57 & 3.3 \\
\hline 2001 & 127491 & 74432 & 73025 & 0.57 & 3.6 \\
\hline
\end{tabular}

Fuente: Elaborado con base en Juan González y Liu Xue Dong, op. cit., p. 629 y Banco Asiático de Desarrollo, Key Indicators of Developing Asian and Pacific Countries, 2002.

les y a la rigidez de la demanda más que a factores políticos, como en el pasado. En ese sentido, podemos afirmar que dicho desempleo depende de criterios estrictamente económicos, aún en la EPE y en las formas de organización comunitaria de la agricultura.

En general, la evolución del empleo ha estado enmarcada en el contexto global de cambio estructural e institucional de la economía. La gran cualidad que ha presentado el mercado laboral es que ha generado empleo formal e informal a la PEA. Es decir, se han generado fuentes de trabajo que se ajustan a las necesidades de la oferta laboral de los trabajadores. Y, aunque la relación entre la tasa de crecimiento de la población y el empleo ha aumentado, el desempleo, sigue presente y más aún, en crecimiento, aunque ahora responde a factores estructurales. Esto ha hecho crecer las cifras de desempleo absoluto, como en 2003, las cuales oscilan entre 24 y 27.5 millones de personas. ${ }^{6}$

6 De acuerdo con el Ministerio de Trabajo y Seguridad Social, 24 millones de personas están buscando trabajo: 10 milones de crecimiento natural de la PEA, 6 millones de despedidos de las EPE y 8 millones desempleados rezagados buscando empleo. Fuente: $h t t p: w w w /$ china.org.cn/ newsandreport 
Cuadro 4

Número de personas empleadas y composición del empleo

(millones de personas y porcentaje)

\begin{tabular}{|c|c|c|c|c|c|c|c|c|c|c|}
\hline & 1980 & 1985 & 1990 & 1995 & 1996 & 1997 & 1998 & 1999 & 2000 & 2001 \\
\hline $\begin{array}{l}\text { Total de } \\
\text { personas } \\
\text { empleadas } \\
\text { Sector }\end{array}$ & 423.6 & 498.7 & 639.1 & 679.5 & 688.5 & 696.2 & 699.6 & 705.9 & 720.8 & 730.2 \\
\hline $\begin{array}{l}\text { Primario } \\
\text { Sector }\end{array}$ & 291.2 & 311.3 & 341.2 & 354.7 & 347.7 & 347.4 & 348.4 & 353.6 & 360.43 & 365.2 \\
\hline $\begin{array}{l}\text { Industrial } \\
\text { Sector }\end{array}$ & 77.36 & 83.5 & 96.9 & 156.3 & 161.8 & 164.9 & 164.4 & 162.4 & 162.2 & 162.8 \\
\hline Servicios & 55.08 & 103.9 & $\begin{array}{r}155.1 \\
\end{array}$ & $\begin{array}{r}168.5 \\
\text { mposici }\end{array}$ & $\begin{array}{c}179.1 \\
\text { n del em }\end{array}$ & $\begin{array}{l}183.7 \\
\text { eo }(\%)\end{array}$ & 186.8 & 189.9 & 189.3 & 202.3 \\
\hline $\begin{array}{l}\text { Sector } \\
\text { primario } \\
\text { Sector }\end{array}$ & 68.70 & 62.4 & & 52.2 & 50.5 & 49.9 & 49.8 & 50.1 & 50.0 & 50.0 \\
\hline $\begin{array}{l}\text { Industrial } \\
\text { Sector }\end{array}$ & 18.30 & 14.7 & & 23.0 & 23.5 & 23.7 & 23.5 & 23.0 & 22.5 & 22.3 \\
\hline Servicios & 13.00 & 22.9 & & 24.8 & 26.0 & 26.4 & 26.7 & 26.9 & 27.5 & 27.7 \\
\hline
\end{tabular}

Fuente: Banco Asiático de Desarrollo (2002) y China Facts and Figures 2002, http:www. china.org.chinadaily.cn

Por otra parte, en 1980, con el cambio en la estructura del mercado laboral, la participación de la empresa privada y transnacional empezó a generar fuentes de trabajo importantes (obviamente, en menor proporción a las generadas por el Estado y el sector agrícola). En lo que corresponde al papel del sector externo, se observa que todavía no es determinante en la transformación estructural, en tanto oferente de empleo, a pesar del gran crecimiento que ha registrado. La aportación de este sector ha sido: 30 mil empleos en 1980; 2 millones en 1990; 12 millones 600 mil empleos en 1994, 16 millones en 1996; 17 millones en 1997, 17.5 millones en 1998 y 20 millones en 2000. Cantidades que representaron el $0.007 \%$ en $1980 ; 2.28 \%$ en 1994 y $2.5 \%$ del total nacional entre 1995 y 2000.

Del análisis realizado hasta ahora, es importante remarcar la transformación que se está generando en la economía, incluyendo sectores tradicionales como el agrícola (véase Cuadro 4). Pese a que China sigue siendo un país dominado por una población rural, lo que explica que, de los 730 millones de empleos existentes hacia 2002, 490 millones $(67.1 \%$ del total) se ubicó en las zonas rurales y los restantes 240 millones en ciudades y poblados.

Un rasgo que empieza a acentuarse es el relacionado con la emergencia del sector servicios como generador de empleo. Pues, aunque el sector agrícola es el principal generador, parece que el de servicios pronto lo desplazará, lo que volvería coherente la estructura productiva (dominada por el sector terciario), con el empleo ofrecido en dicho sector, el 
cual encabezará plenamente la transformación estructural de la economía china en este inicio del siglo XXI.

Por otro lado, la perspectiva de empleo para toda la vida, prevaleciente bajo el socialismo, ha cambiado radicalmente. Hoy día, privan principalmente los criterios de productividad y competitividad. Aunque, como ya dijimos, el bajo costo de la mano de obra, aún le permite captar importantes excedentes laborales y ofrecer empleo a la población desplazada de las empresas rurales y estatales así como a la creciente fuerza de trabajo que año con año se incorpora al mercado laboral (González, 2001). Por ejemplo en 2002, la generación de empleo, desde el punto de vista de la estadística oficial, se comportó acorde a los estándares internacionales, con un crecimiento global durante el periodo de $3.6 \%$.

Como se observa, según las cifras oficiales de empleo, China, de alguna manera está resolviendo su problemática laboral. Aunque siendo un poco más rigurosos, las cifras reportadas de desempleo, que es la otra cara de la moneda, no reflejan del todo la realidad laboral. En efecto, de acuerdo con González y Liu Xue (1998), a las cifras de las agencias oficiales del Estado, respecto a la tasa de desempleo abierto, hay que agregar el disfrazado que aún existe en gran parte de la estructura productiva de ese país, tanto en las EPE como en las empresas rurales. Particularmente en éstas últimas se podría generar un desempleo que afectaría entre 100 y 130 millones de trabajadores si se aplicaran totalmente los criterios de mercado; 27.5 millones de obreros expulsados de las EPE en el periodo 1995-2003. ${ }^{7}$ En términos globales, estas cifras, reflejan gran parte de los conflictos del empleo-desempleo que China debe resolver.

Lo anterior ha llevado a sintetizar la retroalimentación del fenómeno en ocho factores: reforma de las EPE, mejora de la estructura económica, presión demográfica; opciones mutuas entre buscadores de empleo y patrones, insatisfactoria implementación de las políticas de empleo, concepto anticuado sobre éste, impacto de China en la omc y emergencias, como el caso del síndrome respiratorio agudo severo (SAR) que afectó a China en el primer semestre de 2003. ${ }^{8}$ A nuestro parecer, se generará en las empresas modernas que están incorporando y/o desarrollando tecnologías, que requerirán trabajo con alto grado de especialización, el cual, de momento, se encuentra ausente en la República Popular.

Para concluir esta parte, diremos que, para resolver el problema del empleo-desempleo, el gobierno chino tendrá que sostener el crecimiento del producto con tasas similares a las registradas en la última década, pues únicamente este crecimiento será capaz de darle una respuesta real al dato del empleo — el cual ahora ya no sólo está determinado por el Estado sino por el mecanismo de oferta y demanda - . De esta manera, podrá ofrecer oportunidades de empleo, como lo ha hecho con los 325 millones de trabajadores desde 1978.

De esta cantidad, los programas de reempleo y empresas privadas, nacionales y/ o extranjeras, han ocupado a cerca de 17 millones.

8 Peopledaily, noviembre 13 de 2003, Sección Business. 
Adicionalmente, podrá fortalecer la creación de empleo con programas de reempleo y capacitación para los trabajadores expulsados de las empresas estatales y la nueva población solicitante.

\section{Política de empleo y nivel de vida de la población}

Así como la reforma económica y la apertura externa han transformado las viejas estructuras en la vertiente del socialismo de mercado, la población - aquella en nombre de quien se dice se toman las decisiones y acciones para su mejoramiento-, al margen de los nuevos problemas de desempleo, sí ha reflejado cierta mejoría, en particular en el indicador básico: la pobreza. Ésta se ha reducido a cerca de 3\% de la población total: 30 millones de personas en 2002, cuando al inicio del proceso de reforma y apertura externa, se encontraba en niveles superiores a $30 \%$ de la población total. ${ }^{9}$ Este porcentaje implica alguna mejoría en la variable distribución pues, como se observa en el Cuadro 5, el ingreso per cápita ha registrado una tasa de crecimiento semejante a la del PIB.

En efecto, si observamos algunos de los principales indicadores socioeconómicos, que en cierta medida tienen que ver con la reforma económica y su política de empleo, notamos un incremento en los niveles de bienestar de la población, tanto rural como urbana. El principal indicador que refleja los beneficios de la reforma económica sobre la población trabajadora, además del PIB per cápita, es el salario. El ingreso salarial de ambos sectores ha crecido por encima del nivel inflacionario y ello ha redundado en mayor capacidad de compra, diversificación de los bienes adquiridos e introducción de nuevos tipos de bienes, tanto suntuarios como de larga duración.

En el Cuadro 5, que presenta información para años seleccionados, vemos que el ingreso rural y el urbano se han incrementado y que, aunque el diferencial entre ambos ha aumentado ligeramente (el salario urbano era 2.26 veces mayor que el rural en 1979; 2.71 veces en 1995 y 2.78 veces en 2000), han registrado un nivel semejante de crecimiento. Dicha reducción de la pobreza (que en este caso es extrema), como ya dijimos, registra cifras sin parangón en la historia económica mundial del siglo xx.

Si ampliamos la información sobre algunas de las más importantes ciudades y hogares chinos, al menos para el año 2002, observamos una situación más realista en términos de empleo e ingreso, los dos indicadores que estamos considerando como fundamentales para evaluar la eficacia de las políticas de reforma económica y apertura externa.

En efecto, al analizar la información del Cuadro 6, nos damos una idea más real en torno al ingreso per cápita disponible mensual por familia en las ciudades más importantes

9 La línea de la pobreza en China, establecida en términos de ingreso es de 640 RM B o 77.5 dólares anuales. Éstees el dato que se debe tener en mente cuando nos refiramos al abatimiento de la pobreza en China y no a las recomendaciones de la ONU-PNUD. 
Cuadro 5

PIB e indicadores de ingreso e inflación en China, 1979-2002 ${ }^{10}$

(años seleccionados)

\begin{tabular}{ccccccc}
\hline & $\begin{array}{c}\text { PIB (miles de } \\
\text { millones } \\
\text { de dólares, } \\
\text { USA) }\end{array}$ & $\begin{array}{c}\text { PIB } \\
\text { per cápita } \\
\text { (dólares, USA) }\end{array}$ & $\begin{array}{c}\text { Ingreso } \\
\text { Urbano } \\
(\text { en RMB })\end{array}$ & $\begin{array}{c}\text { Ingreso } \\
\text { Rural } \\
\text { (en RMB) }\end{array}$ & $\begin{array}{c}\text { Pobreza } \\
\text { Inflación } \\
\text { (INPC) }\end{array}$ & $\begin{array}{c}\text { (millones } \\
\text { de } \\
\text { personas) }\end{array}$ \\
\hline 1979 & 257.3 & 263.7 & 363 & 160 & 2.6 & 338 \\
1980 & 300.0 & 301.2 & 477 & 191 & 5.9 & 320 \\
1985 & 291.1 & 272.1 & 739 & 397 & 2.2 & 290 \\
1990 & 369.9 & 320.7 & 1510 & 686 & 3.5 & 250 \\
1995 & 707.2 & 593.2 & 4283 & 1577 & 17.1 & 82 \\
1996 & 825.3 & 677.5 & 4838 & 1929 & 8.3 & n.d. \\
1997 & 900.8 & 731.7 & 5160 & 2090 & 2.8 & n.d. \\
1998 & 960.2 & 766.9 & 5425 & 2162 & 0.8 & 45 \\
1999 & 1028.4 & 812.3 & 5854 & 2210 & -1.3 & n.d. \\
2000 & 1077.1 & 850.9 & 6280 & 2253 & -1.4 & 35 \\
2001 & 191.9 & 970.8 & 7703 & 2476 & 0.7 & 30 \\
\hline
\end{tabular}

Fuente: Banco Asiático de Desarrollo 2002, Key Indicators of Developing Asia and Pacific Countries: China People's Republic, pp. 124-134, y Comunicado Estadístico del Buró de Estadísticas del Estado, varios años.

de China. Así, vemos que los habitantes de Beijing, cuentan con un ingreso de 968 RMB mensuales; los de Shanghai de 1 035; los de Hangzhou de 813; los de Ningbo de 922, los de Xiamen, 867; los de Guangzhou, de 1013 y los que mayor ingreso tienen, son los de Shenzhen, con 1617 RMB.

Estos mayores ingresos y empleos son los que han permitido a China reducir los niveles de pobreza e incrementar, por extensión, los de bienestar.

En general, el mayor nivel de vida le ha permitido a la población, ampliar su consumo de bienes materiales e inmateriales. Particularmente, le está permitiendo cambiar la estructura de consumo (Ley de Engel), pues ya no dedica la mayor parte de dicho ingreso a la adquisición de bienes salario, y aun dentro de ésta, existe una diversificación de básicos por carnes, aves, mariscos, hortalizas, frutas y demás. Ahora, la población tiene acceso a un mayor número de bienes como son: casas, coches, electrodomésticos, computadoras, relojes, joyas, turismo interno y externo y, en general, bienes de consumo masivo.

\section{Regreso-admisión al GATT-OMC y desafío para el mercado laboral chino}

Antes de iniciar este apartado, es necesario realizar un breve recordatorio en torno al asunto de China y el GATT-OMC. China, participó en las negociaciones de creación del GATT

10 Los datos aquí consignados fueron publicados en otras fuentes como la Comisión Económica y Social para Asia Pacífico; las estadísticas financieras del Fondo M onetario Internacional, Banco de China y el Banco Mundial en su último informe sobre desarrollo humano 2002 en China, entre otros. 
Cuadro 6

Condiciones básicas de los hogares urbanos de 35 ciudades chinas, 2002

\begin{tabular}{|c|c|c|c|c|c|c|}
\hline Región & $\begin{array}{c}\text { Hogares } \\
\text { encuestados } \\
\text { (hogares) }\end{array}$ & $\begin{array}{c}\text { Promedio } \\
\text { de personas } \\
\text { por hogar } \\
\text { (hogares) }\end{array}$ & $\begin{array}{c}\text { Promedio de } \\
\text { empleados } \\
\text { por hogar } \\
\text { (personas) }\end{array}$ & $\begin{array}{c}\text { Número } \\
\text { promedio de } \\
\text { dependientes } \\
\text { por empleado } \\
\text { (personas) }\end{array}$ & $\begin{array}{c}\text { Ingreso } \\
\text { mensual } \\
\text { per cápita } \\
\text { (yuan) }\end{array}$ & $\begin{array}{c}\text { Ingreso } \\
\text { disponible } \\
\text { mensual } \\
\text { per cápita } \\
\text { (yuan) }\end{array}$ \\
\hline Promedio & 13100.00 & 3.00 & 1.53 & 1.96 & 791.88 & 735.86 \\
\hline Beijing & 1000.00 & 2.98 & 1.64 & 1.82 & 1040.50 & 968.98 \\
\hline Tianjin & 1500.00 & 3.03 & 1.46 & 2.08 & 723.95 & 682.77 \\
\hline Shijiazhuang & 400.00 & 2.99 & 1.46 & 2.04 & 622.72 & 587.49 \\
\hline Taiyuan & 300.00 & 2.95 & 1.48 & 1.99 & 616.72 & 582.77 \\
\hline Hohhot & 400.00 & 2.78 & 1.32 & 2.11 & 562.72 & 547.81 \\
\hline Shenyian & 500.00 & 3.04 & 1.80 & 1.69 & 637.17 & 587.34 \\
\hline Dalian & 500.00 & 3.03 & 1.66 & 1.83 & 672.53 & 629.72 \\
\hline Changchun & 300.00 & 3.07 & 1.75 & 1.75 & 603.89 & 572.05 \\
\hline Harbin & 500.00 & 2.89 & 1.45 & 2.00 & 613.75 & 585.59 \\
\hline Shanghai & 500.00 & 2.91 & 1.52 & 1.91 & 1120.16 & 1035.30 \\
\hline Ninjing & 300.00 & 2.90 & 1.60 & 1.81 & 779.31 & 721.52 \\
\hline Hangzhou & 300.00 & 2.97 & 1.52 & 1.96 & 912.61 & 813.20 \\
\hline Ningbo & 200.00 & 3.01 & 1.74 & 1.73 & 1038.77 & 922.61 \\
\hline Hebei & 200.00 & 2.95 & 1.53 & 1.93 & 585.16 & 549.58 \\
\hline Fuzhou & 300.00 & 3.10 & 1.64 & 1.90 & 692.14 & 639.81 \\
\hline Xiamen & 200.00 & 3.22 & 1.78 & 1.81 & 946.18 & 867.95 \\
\hline Nanchang & 300.00 & 2.99 & 1.55 & 1.93 & 566.64 & 552.21 \\
\hline Jinan & 300.00 & 3.01 & 1.62 & 1.86 & 832.30 & 774.52 \\
\hline Qingdao & 400.00 & 2.88 & 1.44 & 2.00 & 750.76 & 674.30 \\
\hline Zhengzhou & 400.00 & 2.9 & 1.5 & 2.34 & 720.61 & 684.02 \\
\hline Wuhan & 500.00 & 3.06 & 1.45 & 2.11 & 648.44 & 613.77 \\
\hline Changsha & 200.00 & 3.02 & 1.56 & 1.93 & 680.25 & 633.44 \\
\hline Guangzhou & 300.00 & 3.15 & 1.64 & 1.93 & 1163.25 & 1013.57 \\
\hline Shenzhen & 100.00 & 3.23 & 1.79 & 1.80 & 1711.34 & 1617.22 \\
\hline Nanning & 200.00 & 3.00 & 1.60 & 1.87 & 719.06 & 645.58 \\
\hline Haikou & 200.00 & 3.46 & 1.59 & 2.17 & 636.61 & 606.11 \\
\hline Chengdu & 300.00 & 3.03 & 1.45 & 2.08 & 624.42 & 593.12 \\
\hline Chongging & 300.00 & 2.93 & 1.32 & 2.22 & 791.49 & 742.21 \\
\hline Guiyang & 200.00 & 3.20 & 1.45 & 2.21 & 577.07 & 563.03 \\
\hline Kunming & 300.00 & 2.92 & 1.43 & 2.04 & 624.65 & 588.86 \\
\hline Lasa & 0.00 & 0.00 & 0.00 & 0.00 & 0.00 & 0.00 \\
\hline$X^{\prime}{ }^{\prime} a n$ & 300.00 & 3.02 & 1.48 & 2.04 & 618.80 & 574.27 \\
\hline Lanzhou & 300.00 & 2.98 & 1.45 & 2.06 & 565.96 & 539.04 \\
\hline Xining & 300.00 & 2.96 & 1.18 & 2.51 & 590.98 & 561.43 \\
\hline
\end{tabular}

Fuente: Tomado de China Statistical Date, en http:www.china.org.cn/e-company

(Aquino Rodríguez, 1998), firmó su adhesión al mismo el 30 de octubre de 1947 y depositó en mayo de 1948 los Instrumentos de Aceptación y de Aplicación Provisional. Actuó como país parte contratante, por ello, se le reconoce como fundador de la organización en 1948. Pero, con el triunfo de la revolución socialista en 1949, China continental y no Taiwán, notificó en marzo de 1950 su retiro (González; 2001:200). A partir de entonces, China perdió contacto directo con el GATT, quedando el asunto indefinido hasta que en julio de 1986, justo antes del inicio de la Ronda Uruguay (septiembre del mismo año 
en Punta del Este, Uruguay), intentó regresar al organismo rector del comercio mundial en tanto socio fundador. ${ }^{11}$

Después de ser rechazada su propuesta de reingresar al GATT, éste, creó un grupo de negociación en marzo de 1987 para analizar la solicitud junto con una delegación china. Ambos se reunieron en octubre de ese año y, a partir de entonces, inició el proceso que concluyó en diciembre de 2001 al admitir la OMC la solicitud china.

Nosotros planteamos que este proceso se inició con el GATT, organismo del que China fue país parte contratante y que es, en el mejor de los casos, un doble proceso: regreso al GATT-admisión a la OMC, pues si bien, esta última es una organización reconocida formalmente por los 146 miembros a abril de 2003, según sus principios y declaratoria de creación (OMC, 2003), es el GATT, más los nuevos temas comerciales en el intercambio de bienes y servicios, derivados de la última ronda de negociaciones, quien le dio nacimiento en la Ronda Uruguay. Fue en el gatT donde se inició y — de acuerdo con los temas negociados y los plazos para la implantación de los principales acuerdos (sobre derechos de propiedad intelectual, barreras no arancelarias, agricultura, subvenciones, solución de diferencias, servicios) por China-, es al GATT a quien le correspondió avalar, después de quince años de negociaciones (ocho en el GATT y siete en la OMC) la solicitud china.

Por los plazos establecidos en la negociación final para la apertura de su economía, planteamos que se impuso la visión china de ser admitida como nación subdesarrollada ${ }^{12}$ pues la mayoría de sus compromisos se irán cumpliendo gradualmente y su apertura comercial y financiera se realizará hasta 2005, tal y como se negoció con Estados Unidos en 1999 y la Unión Europea en 2000 (González, 2001). ${ }^{12}$

11 Uno de los factores que obstaculizaron la intención de la República Popular de reasumir su lugar en tanto país parte contratante fundador del GATT en 1948 fue precisamente el estatus de integrante originario, que siempre antepuso ante el GATT en julio de 1986. De hecho, en el fondo, quitando todo factor político de los últimos cinco años previos a la admisión, la esencia del debate en torno a ésta se relaciona, desde nuestro punto de vista, con dicha circunstancia. Es decir, al ser admitida China en el organismo, asumiría los ben eficios de dicho estatus, en tanto país en desarrollo. Esto, finalmente lo logró, si observamos los plazos de desgravación arancel aria negociados con la O M C para 2005. En dicho año, que conicide con su propio programa de liberación, tendrá un nivel arancelario en bienes y ser vicios semejante a los países subdesar rollados miembros del organismo. Es decir, ocho años previos de negociación son el antecedente de la última fase, a partir de la creación de la OM C en 1995, fase sin la cual no podría entenderse el desenlacefinal (al respecto, véase Juan González García, “La política comercial de la RPC y su aceptación regreso a la OMC" en Estudios del Pacífico, núm. 4, CEAA y PE-APEC, M éxico, El Colegio de M éxico, 2001; y del mismo autor, "El ingreso de China a la OM C: un desafío en puerta, Comercio Exterior, núm. 5, vol. 52, México, 2001, pp. 454-462.

12 De hecho, la discusión acerca del status quo del grado de desarrollo económico de China parecería que pasó a segundo término, pues sólo se realizó en las negociaciones iniciales. En las posterior es, el debate se centró, principalmente, en los nuevos temas o por los emanados de la Ronda Uruguay y de los compromisos asumidos tanto por los países desarrollados como subdesarrollados en las negociaciones de Marruecos en 1994, durante la aprobación y creación de la OMC. 
El grueso de los compromisos en los sectores arancelario (10\% hacia 2005), agrícola, industrial, comercial, de servicios, gobierno, propiedad intelectual e industrial, sistema financiero (bancario, seguros, cambiario) están directamente relacionados con el laboral. Debido a que, al adoptar China las normas del comercio internacional, tendrá que terminar de modificar su estructura institucional y liberar sus diversos mercados. Lo que en pocas palabras significa finalizar con la protección del sistema económico chino heredado del socialismo y ello redundará, en última instancia, en la mayor liberación del mercado laboral y el predominio del mecanismo de mercado en la asignación del factor trabajo.

De hecho, algunos escenarios pronosticaron — y pronostican — efectos positivos sobre la economía china, derivados de su admisión al organismo en términos de crecimiento del PIB, transferencia y desarrollo de tecnología, aumento de ingresos per cápita y de las regiones, incremento del empleo, montos record de IED, crecimiento del comercio externo, aumento del consumo privado, entre otros (Banco Mundial, 1998). Tan sólo medido en términos del PIB, se esperaba que el regreso lo impactara entre 0.7 y $1.2 \%$ con lo que se podrían crear entre 700 mil y un millón doscientos mil empleos.

Algunos otros autores como Aquino Rodríguez $(1998,1999)$ plantean que la admisión de China a la омc le generará problemas, especialmente en los sectores, industrias y regiones tradicionales y atrasadas, además de alrededor de 300 millones de personas que viven en la miseria absoluta.

En lo referente a la política de empleo y la evolución de este sector, tenemos lo siguiente: China se comprometió ante la OMC a eliminar los límites al movimiento del trabajo entre las áreas rurales y urbanas, con lo que abolirá el sistema de registro de residencia, impactando con ello las políticas migratorias, pues se permitirá el libre flujo de trabajadores, particularmente de las áreas rurales. Con ello, se facilitará la creación de un mercado laboral unificado y éste se guiará por la oferta y la demanda.

Por lo que respecta a la evolución del empleo, la misma fuente nos indica que se han creado cerca de 8 millones, cifra insuficiente para las necesidades de alrededor de 17 millones de trabajadores desempleados. Así, la tasa de desempleo se ha elevado a alrededor de $3.8 \%$, esperándose un incremento hasta $4.2 \%$ al finalizar 2003. Tasa que representa —después de $4.5 \%$ de 2002 — la segunda mayor de las últimas dos décadas.

Por ejemplo, en 2002, las EPE expulsaron 6.5 millones de trabajadores y las empresas rurales alrededor de 10 millones; del total, sólo se reemplearon 790 mil trabajadores. El problema, es que los planes de reempleo vienen a la baja, pues si bien en 1998 se atendían $50 \%$ de las solicitudes de empleo, para 2001 sólo se pudo atender $30 \%$.

Como ya hemos mencionado, para atender al problema del empleo, China deberá mantener una alta tasa de crecimiento del PIB ( $8 \%$ de acuerdo con las autoridades). Pero sí sabemos que, en promedio, durante el periodo 2002-2006 se crearán cerca de 50 millones de empleos y también que, aún con un crecimiento del comercio internacional chino con 
tasas elevadas (como efecto de una mayor apertura externa de la economía internacional y de su regreso al GATT-OMC), podrán crearse entre 750 mil y dos millones de empleos adicionales, también, por año entre 2002 y 2005, creándose 10 millones de empleos nuevos al año, por lo que la cifra óptima será de 60 millones en los próximos cinco años.

Ahora bien, desde el punto de vista de nuestras hipótesis de trabajo, la ventaja de esta nueva situación del mercado laboral se regirá por las leyes del mercado. La empresa pública, privada o social buscará ella misma; los trabajadores tendrán que competir por los pocos puestos que se crearán (pocos únicamente por tratarse del país más poblado del mundo, pues con seguridad, no habrá país en el mundo que tenga la capacidad de generar los empleos que eventualmente China pudiera crear en los próximos cinco años). ${ }^{13}$ Los que no entren tendrán que capacitarse en las nuevas actividades que, preponderantemente, generará el sector servicios (en los pasados cinco años, generó 87\%, en su defecto, deberán emigrar a los países de ultramar que cuentan con colonias de chinos. El gobierno tendrá el desafío de seguir aplicando una política laboral que facilite y promueva el empleo.

\section{Conclusiones}

Aquí planteamos que sería interesante analizar lo que cada país está haciendo para atender la problemática internacional del empleo-desempleo.

Con este panorama como antecedente, grosso modo, se analizó la política, evolución y estructura del empleo en China en el último medio siglo. Concluimos que, a la par de la transformación de la estructura económica, guiada por la reforma y apertura externa, el mercado laboral se está transformando y la generación de empleo está determinada principalmente por el mecanismo de mercado en el que priva el factor productividad para la colocación de trabajadores más que por criterios políticos; por el comportamiento del sector servicios; y por las políticas del gobierno para facilitar y promover la contratación por la empresa y la colocación por los trabajadores.

Derivado de nuestro análisis, afirmamos que China, de una u otra manera, está afrontando con relativo éxito el problema del empleo-desempleo, a pesar de que existen factores en el corto plazo que difícilmente permitirán su resolución inmediata. El gobierno ha desempeñado un papel determinante para favorecer y facilitar el crecimiento económico, que hasta ahora ha sido el mejor antídoto para atacar el problema del empleo.

No obstante la problemática subyacente del mercado laboral, creemos que con la reforma y la apertura económica, previo doloroso proceso de aprendizaje, se han sentado las bases para la normalización del mercado laboral, donde los criterios de mercado, utilidad,

13 De acuerdo a Ping Xinqiao y Fan Ying (2003), la competencia por el empleo se está dando en todas las áreas de la economía y está favoreciendo el factor capacidades, destrezas, escolaridad y experiencia. Ello, incide directamente en la productividad del trabajo y en su remuneración. 
capacidades, aptitudes, información y libre movilidad interna y de conocimiento, tenderán a sustituir y eliminar, de una vez por todas, las viejas prácticas del socialismo. Lo que no significa afirmar que el Estado deje de cumplir la función preponderante que hasta ahora ha desempeñado al determinar la política de empleo.

Solo restaría esperar que el sistema económico no sufriera los embates de crisis económicas o políticas recurrentes en el escenario internacional que pusieran en peligro los débiles equilibrios — que si se llegaran a dar esperemos se den con tasas de desempleo de entre 5 y $8 \%$ - de este mercado y se sienten las bases para su sano desenvolvimiento en el mediano y largo plazos. Para ello se requiere que dicho país mantenga la tasa de crecimiento por un periodo similar al de la reforma de mercado actual.

\section{Bibliografía}

Aaronson, Daniel y Kenneth Housinger, "The Impact of the Technology on Displacement and Reemployment", en Economic Perspectives, núm. 2, vol. 23, 1999.

Aquino Rodríguez, Carlos, "China en la Organización Mundial de Comercio", en http: www.mx. geo cities.com/gunnm_dream/china.omc.html, 1998.

- China en la Organización Mundial de Comercio y la Reunión de Seattle, en http: www.mx. geocities.com/gunnm_dream/china.omc. html, 1999.

Banco Asiático de Desarrollo, Key Indicators of Developing Asia and Pacific Countries: China People's Republic, 2002, pp. 124-134.

- Key Indicators of Developing Asia and Pacific Countries: China People's Republic, 2002, pp. 124-125.

Banco Mundial, "China Engaged. Integration with the Global Economy”, en China 2020 (serie), Washington, DC, 1998.

Cornejo Bustamante, Romer, "China: una revisión de cincuenta años de historia”, en R. Eugenio Anguiano (coordinador), China contemporánea La construcción de un país (desde 1949), México, El Colegio de México, 2001.

China Statistical Date, en http:www.china.org.cn/ecompany, 2003.

Deleyne, Jean, La economía china, México, Planeta, 1976.

Documento: "Situación del trabajo y la seguridad social en China", en Beijing Informa, núms. 21 y 22, abril de 2002, Beijing.

Eckstein, Alexander, China's Economic Revolution, Cambridge University, USA, 1977.
Fang, Shan, "The Evolution of Labor Policy in Mainland China", en Issues and Studies, núm. 5, vol. 32, Taiwán, Republic of China, 1996.

González García, Juan y Liu Xue Dong, "El mercado laboral en China. Situación actual y perspectivas", en Comercio Exterior, núm. 7, vol. 49, México.

, "La República Popular China a finales del siglo xx, Logros y desafíos hacia el siglo XxI", en Problemas del Desarrollo, Mexico, UNAM-IIEC, 2001, pp. 171-205.

_ , "La Relación China-Unión Europea en el marco de la Organización Mundial del Comercio”, en Joaquín Roy y Roberto Domínguez Rivera (coordinadores), Las relaciones exteriores de la Unión Europea, México, unAM-Plaza y Váldes, 2001, pp. 307-321.

Muqiao, Xue, Problemas de la economía socialista de China, Beijing, Lenguas Extranjeras, 1982.

OECD, "Labor Marquet and Social Benefits Policies" en China in the World Economy (The Domestic Plicy Challenges), París, 2002, pp. 537-577.

OIT, Informe sobre el Empleo en el Mundo 1998 1999. Tendencias del Empleo en el Mundo: Un Panorama Desalentador, 2000, p. 7. , World Employment Report 2001, p. 2, (Overview).

OMC, "Para Entender a la oMC", en $h t t p: / / W w W$. wto.org/

Rodríguez y Rodríguez, Ma. Teresa, Industrialización de Zonas Rurales en China, UNAM-FE, Investigación Económica, México, 1998.

State Statistical of Bureau. Yearbook, 1993, 1998 y 2002. 
Xinqiao Ping y Fan Ying, Wage Contract, Gray Income and Career Concern, Beijing, Pekin University, Center for Economic Research, 2003.
Zhong Zhao, Migration, Labor Market Flexibility, and Wage Determination in China. A review, Beijing, JEL, Pekin University, 2003. 